\title{
On a Serrin-type regularity criterion for the Navier-Stokes equations in terms of the pressure
}

\section{Journal Article}

Author(s):

Struwe, Michael

Publication date:

2007

Permanent link:

https://doi.org/10.3929/ethz-b-000003956

Rights / license:

In Copyright - Non-Commercial Use Permitted

Originally published in:

Journal of Mathematical Fluid Mechanics 9(2), https://doi.org/10.1007/s00021-005-0198-y 


\title{
On a Serrin-Type Regularity Criterion for the Navier-Stokes Equations in Terms of the Pressure
}

Michael Struwe

Communicated by $Y$. Giga

\begin{abstract}
We prove a Serrin-type regularity result for Leray-Hopf solutions to the Navier-
\end{abstract} Stokes equations, extending a recent result of Zhou [28].

Mathematics Subject Classification (2000). 35B65, 35Q30, 76D05.

Keywords. Navier-Stokes equations, regularity criteria.

\section{Conditional regularity results for the Navier-Stokes system}

Let $\Omega$ be a domain in $\mathbb{R}^{n}, n \geq 3$, and let $T>0$. On the space-time region $\Omega \times[0, T]$ we consider the Navier-Stokes equations

$$
\frac{\partial u^{i}}{\partial t}+u \cdot \nabla u^{i}=\Delta u^{i}-\frac{\partial p}{\partial x^{i}}, i=1, \ldots, n,
$$

together with the divergence condition

$$
\operatorname{div} u=\sum_{i=1}^{n} \frac{\partial u^{i}}{\partial x_{i}}=0,
$$

for a velocity field $u=\left(u^{1}, \ldots, u^{n}\right)$ and an associated pressure function $p$.

Given any smooth initial velocity distribution $u_{0}$ vanishing on $\partial \Omega$ and imposing the boundary condition

$$
u=0 \text { on } \partial \Omega \times[0, T],
$$

we seek to find a smooth solution $(u, p)$ of (1)-(3) for all $T>0$ such that $u=u_{0}$ at $t=0$. By work of Leray [15] and Hopf [12] there always exists a global weak solution $u \in L^{\infty}\left(\left[0, \infty\left[; L^{2}(\Omega)\right) \cap L^{2}\left(\left[0, \infty\left[; H_{0}^{1}(\Omega)\right)\right.\right.\right.\right.$ to this problem. However, in contrast to the case when $n=2$, where the global existence of a smooth solution was shown by Ladyzhenskaya [13], [14], for $n \geq 3$ so far a smooth solution is only known to exist for short time. On the other hand, it is well-known that the 
Leray-Hopf solution coincides with the smooth solution as long as the latter exists. It is therefore conjectured that the Leray-Hopf solution actually is smooth and provides the desired global extension of the known local classical solution. The existence problem is thereby substituted by the problem of proving regularity of weak solutions of the Leray-Hopf type. The situation is similar in the case when $\Omega=\mathbb{R}^{n}$ or in the spatially periodic case where we consider only (1), (2) with smooth data $u=u_{0}$ at $t=0$ either having compact support or being spatially periodic.

Throughout the remainder of this paper we focus on the case when $\Omega=\mathbb{R}^{n}$ or when $u$ is spatially periodic.

As was first shown by Serrin [18], under certain additional hypotheses a LerayHopf solution indeed is regular; independently, this was also shown by Ohyama [16]. To state this result we introduce the space

$$
L_{x, t}^{r, s}=L^{s}\left([0, T] ; L^{r}(\Omega)\right)
$$

with norm

$$
\|u\|_{L_{x, t}^{r, s}}=\left(\int_{0}^{T}\|u(\cdot, t)\|_{L^{r}(\Omega)}^{s} d t\right)^{1 / s} .
$$

Theorem 1.1. Suppose $u \in L^{\infty}\left([0, T] ; L^{2}(\Omega)\right) \cap L^{2}\left([0, T] ; H_{0}^{1}(\Omega)\right)$ is a weak solution of the Navier-Stokes system (1)-(3), and assume in addition that $u \in L_{x, t}^{r, s}$ where $\frac{n}{r}+\frac{2}{s} \leq 1$. Then $u$ is smooth.

In Serrin's original work the slightly stronger condition $\frac{n}{r}+\frac{2}{s}<1$ is imposed. The above improvement is due to Fabes-Jones-Riviere [9], Giga [11], Sohr [19], and von Wahl [25]; the corresponding local regularity result of Serrin was extended to the limit case by Struwe [22] and Takahashi [23], [24]. In the case $r=n=3$, $s=\infty$ at first an additional smallness assumption was required which was recently removed by Escauriaza-Seregin-Šverák [8], [17]. Further references can be found, for instance, in [5].

A number of authors have studied similar conditional regularity results involving either the pressure, or combinations of $u$ and $p$; see for instance Beirão da Veiga [2]-[4], Chae-Lee [7], or Berselli-Galdi [5]. Note that a rough dimensional analysis as in Caffarelli-Kohn-Nirenberg [6] predicts the regularity of weak solutions to (1)-(3) provided either the condition

$$
\nabla p \in L_{x, t}^{r, s} \text { with } \frac{n}{r}+\frac{2}{s} \leq 3
$$

or the condition

$$
p \in L_{x, t}^{r^{*}, s} \text { with } \frac{n}{r^{*}}+\frac{2}{s} \leq 2
$$

is satisfied.

The following result is due to Berselli-Galdi [5], Theorem 1.1 and Theorem 3.3; some technical improvements were later obtained by Zhou [26]. 
Theorem 1.2. Suppose $u \in L^{\infty}\left([0, T] ; L^{2}\left(\mathbb{R}^{n}\right)\right) \cap L^{2}\left([0, T] ; H_{0}^{1}\left(\mathbb{R}^{n}\right)\right)$ is a weak solution of the Navier-Stokes system (1), (2) with associated pressure $p$. Also assume that one of the following conditions is satisfied:

i) The pressure satisfies (5) with $1 \leq s<\infty$.

ii) The pressure gradient satisfies condition (4) with $1 \leq s \leq n$. Then $u$ is smooth.

Berselli-Galdi remark that one obtains regularity also in the limit case $p \in$ $L_{x, t}^{n / 2, \infty}$, provided the corresponding norm is sufficiently small; see [5], Remark 1.3.

In the case when the spatial dimension is at most 4, Zhou [27], [28] recently was able to remove the restriction on $s$ in the second part of Theorem 1.2. Here, at least for finite values of $r$, we extend his result to arbitrary dimensions $n \geq 3$.

Theorem 1.3. Let $n \geq 3$. Suppose $u \in L^{\infty}\left([0, T] ; L^{2}\left(\mathbb{R}^{n}\right)\right) \cap L^{2}\left([0, T] ; H_{0}^{1}\left(\mathbb{R}^{n}\right)\right)$ is a weak solution of the Navier-Stokes system (1), (2) with associated pressure $p$. Assume $\nabla p$ satisfies condition (4) with $n / 3<r<\infty, 2 / 3<s<\infty$. Then $u$ is smooth. The same result also holds when $u$ is spatially periodic.

Conceivably, Theorem 1.3 holds true on a general domain. In fact, part ii) of Theorem 1.2 remains valid in this case; see [5], Theorem 3.3. However, similar to Berselli-Galdi [5], Zhou [26], [28] our proof of Theorem 1.3 requires the estimates

$$
\|p\|_{L^{q}} \leq C\|u\|_{L^{2 q}}^{2}, 1<q<\infty
$$

and

$$
\|\nabla p\|_{L^{q}} \leq C|| \mid \nabla u\|u\|_{L^{q}}, 1<q<\infty
$$

which only seem to be available when either $\Omega=\mathbb{R}^{n}$ or in the periodic setting. In these cases these estimates easily follow from the Calderón-Zygmund inequality applied to the equation

$$
-\Delta p=\operatorname{div}(u \cdot \nabla u)=\sum_{i, j=1}^{n} \partial_{i} \partial_{j}\left(u^{i} u^{j}\right)
$$

resulting from (1) and (2). Note, however, that by work of Solonnikov [21] and Sohr - von Wahl [20] space-time estimates analogous to $(6),(7)$ hold on a general domain. In particular, for $u \in L^{\infty}\left([0, T] ; L^{2}\left(\mathbb{R}^{n}\right)\right) \cap L^{2}\left([0, T] ; H_{0}^{1}\left(\mathbb{R}^{n}\right)\right)$ we have bounds for $\nabla p$ in $L_{x, t}^{r, s}$ with exponents satisfying $\frac{n}{r}+\frac{2}{s}=n+1$, and similarly for $p$ in $L_{x, t}^{r^{*}, s}$ with exponents satisfying $\frac{n}{r^{*}}+\frac{2}{s}=n$. By interpolation, therefore, in the following we may always assume that (4) or (5) hold with equality.

\section{Proofs}

Let $u$ be a smooth solution of the Navier-Stokes system (1), (2) on $\mathbb{R}^{n} \times[0, T]$. We propose to establish a-priori bounds that will allow to extend $u$ for all time. 
Upon multiplying equation (1) with $u|u|^{\theta-2}$ for some number $\theta \geq n$ and integrating by parts, thereby observing (2), we obtain the well-known identity

$$
\begin{aligned}
\frac{1}{\theta} \frac{d}{d t} \int_{\mathbb{R}^{n}}|u|^{\theta} d x & +\int_{\mathbb{R}^{n}}|\nabla u|^{2}|u|^{\theta-2} d x+\left.\left.\frac{4(\theta-2)}{\theta^{2}} \int_{\mathbb{R}^{n}}|\nabla| u\right|^{\theta / 2}\right|^{2} d x \\
& =-\int_{\mathbb{R}^{n}} u \cdot \nabla p|u|^{\theta-2} d x=: I(t)
\end{aligned}
$$

for $0<t<T .1$

Let $v=|u|^{\theta / 2}$. Provided that we succeed in bounding the time integral of $I(t)$, equation (8) will yield a bound for $v$ in the space $L_{x, t}^{2, \infty} \cap L^{2}\left([0, T] ; H_{0}^{1}\left(\mathbb{R}^{n}\right)\right)$. By Sobolev's embedding theorem then $v$ also is bounded in the space $L_{x, t}^{2, \infty} \cap L_{x, t}^{2^{*}, 2}$, where $2^{*}=\frac{2 n}{n-2}$. By interpolation, finally, $v$ will be bounded in the scale of spaces $L_{x, t}^{p, q}$ for any pair of exponents $p, q$ such that

$$
\frac{n}{p}+\frac{2}{q}=\frac{n}{2}, 2 \leq p \leq 2^{*}, 2 \leq q \leq \infty,
$$

and for any such choice of exponents with a uniform constant $C=C(p, q)$ we have

$$
\|v\|_{L_{x, t}^{p, q}}^{2} \leq C\left(\|v\|_{L_{x, t}^{2, \infty}}^{2}+\|\nabla v\|_{L_{x, t}^{2,2}}^{2}\right) \leq C\left(\left\|v_{0}\right\|_{L^{2}}^{2}+\int_{0}^{T} I(t) d t\right) .
$$

If $\theta \geq n$, now (10) implies that $u \in L_{x, t}^{r, s}$ for any pair of exponents $r, s \geq n$ satisfying the condition $\frac{n}{r}+\frac{2}{s} \leq 1$, and regularity of $u$ follows from Theorem 1.1 . It therefore remains to bound $I(t)$ in terms of $\|v\|_{L_{x, t}^{p, q}}^{2}$ for a suitable choice of $\theta \geq n, p$, and $q$.

Given exponents $r$ and $s$ satisfying (4), we can attempt to bound

$$
I(t) \leq \int_{\mathbb{R}^{n}}|\nabla p \| u|^{\theta-1} d x
$$

and then

$$
\begin{aligned}
\int_{0}^{T} I(t) d t & \leq \int_{0}^{T} \int_{\mathbb{R}^{n}}|\nabla p \| u|^{\theta-1} d x d t \\
& \leq\left.\|\nabla p\|_{L_{x, t}^{r, s}\|u\|_{L_{x, t}^{(\theta-1) r^{\prime},(\theta-1) s^{\prime}}}^{\theta-1} \leq\|\nabla p\|}\right|_{L_{x, t}^{r, s}\|v\|_{L_{x, t}^{p, q}}^{2 \frac{\theta-1}{\theta}},}
\end{aligned}
$$

where $r^{\prime}$ and $s^{\prime}$ are conjugate to $r$ and $s$, respectively, with

$$
1=\frac{1}{r}+\frac{1}{r^{\prime}}, 1=\frac{1}{s}+\frac{1}{s^{\prime}}
$$

and where $\theta$ is chosen so that the exponents

$$
p=2 \frac{\theta-1}{\theta} r^{\prime}, q=2 \frac{\theta-1}{\theta} s^{\prime}
$$

\footnotetext{
1 The idea of using powers of the unknown function $u$ as testing functions already appears around 1960 in the work of Moser on Harnack's inequality for elliptic and parabolic equations. Not surprisingly, also equation (8) in the context of the Navier-Stokes equations is known since the 1970's. It explicitly appears, for instance, in the the work of Galdi-Rionero [10] or Beirão da Veiga [1].
} 
satisfy (9). Note that the conditions $2 \leq p \leq 2^{*}, 2 \leq q \leq \infty$ impose the constraints

$$
\frac{n \theta}{n+2 \theta-2} \leq r \leq \theta, 1 \leq s \leq \theta
$$

on any admissible choice of $r$ and $s$. Moreover, equations (9) and (13) yield

$$
\frac{n}{r}+\frac{2}{s}=(n+2)-\left(\frac{n}{r^{\prime}}+\frac{2}{s^{\prime}}\right)=(n+2)-2 \frac{\theta-1}{\theta}\left(\frac{n}{p}+\frac{2}{q}\right)=2+\frac{n}{\theta}
$$

and equation (4) implies $\theta=n$.

The second assertion in Theorem 1.2 now follows easily. Observe that we do not use either (6) or (7).

Proof of Theorem 1.2.ii) [5]. Choosing $\theta=n$, for any pair of numbers $r$ and $s$ satisfying the conditions (4) and (14), equation (13) defines an admissible pair $p, q$ satisfying (9). We then may use equation (10) and (12) to obtain

$$
\|v\|_{L_{x, t}^{p, q}}^{2} \leq C\left(\left\|v_{0}\right\|_{L^{2}}^{2}+\|\nabla p\|_{L_{x, t}^{r, s}}^{r, \| v} \|_{L_{x, t}^{p, q}}^{2 \frac{n-1}{n}}\right) .
$$

Since $\frac{n-1}{n}<1$, the estimate (15) implies that $\|v\|_{L_{x, t}^{p, q}}^{2}$ is a-priori bounded for any time $T$. As observed above, regularity of $u$ then follows from Theorem 1.1.

Estimate (15) clearly can be improved. Also using the bound (7) with $q=2$ and observing that the term $\|\mid \nabla u\| u \|_{L^{2}}^{2}$ may be absorbed on the left of (8) when $\theta=4$, in [28] Zhou was able to extend the admissible range of exponents $r$ and $s$ to the full range of exponents as given by (4). However, since we require that $\theta \geq n$, this approach only seems to work in dimenions $n \leq 4$.

As an alternative to (11), for $0<t<T, \theta \geq n$ we may also integrate by parts and use (2) to estimate

$$
\begin{aligned}
I(t) & \leq(\theta-2) \int_{\mathbb{R}^{n}}|p||\nabla| u|||u|^{\theta-2} d x \\
& \leq \frac{2(\theta-2)}{\theta}\left(\int_{\mathbb{R}^{n}}|p|^{2}|u|^{\theta-2} d x\right)^{1 / 2}\left(\left.\left.\int_{\mathbb{R}^{n}}|\nabla| u\right|^{\theta / 2}\right|^{2} d x\right)^{1 / 2} .
\end{aligned}
$$

Combining the above two ways of bounding $I(t)$, we can now extend Zhou's result to arbitrary dimension $n \geq 3$.

Proof of Theorem 1.3. We may assume that $r \geq n$. Indeed, by the GagliardoNirenberg inequality for $r<n$ we have $\|p\|_{L_{x, t}^{r^{*}, s}} \leq C\|\nabla p\|_{L_{x, t}^{r, s}}$, with $r^{*}$ satisfying $\frac{1}{r^{*}}=\frac{1}{r}-\frac{1}{n}$. Therefore the case $r<n$ already is covered by Theorem 1.2.i).

For $0<t<T, \theta \geq n$ we use (11) and (16) together with Young's inequality $|a b| \leq 3 \delta^{-4 / 3} a^{4 / 3} / 4+\delta^{4} b^{4} / 4$ for $a, b \in \mathbb{R}, \delta>0$ to estimate, with constants 


$$
\begin{aligned}
& C=C(\theta), \\
& I(t) \leq C\left(\int_{\mathbb{R}^{n}}|\nabla p \| u|^{\theta-1} d x\right)^{1 / 2}\left(\int_{\mathbb{R}^{n}}|p|^{2}|u|^{\theta-2} d x\right)^{1 / 4}\left(\left.\left.\int_{\mathbb{R}^{n}}|\nabla| u\right|^{\theta / 2}\right|^{2} d x\right)^{1 / 4} \\
& \quad \leq C\left(\int_{\mathbb{R}^{n}}|\nabla p \| u|^{\theta-1} d x\right)^{2 / 3}\left(\int_{\mathbb{R}^{n}}|p|^{2}|u|^{\theta-2} d x\right)^{1 / 3}+\left.\left.\frac{2}{\theta^{2}} \int_{\mathbb{R}^{n}}|\nabla| u\right|^{\theta / 2}\right|^{2} d x .
\end{aligned}
$$

Applying Hölder's inequality with exponents $\frac{\theta+2}{4}$ and $\frac{\theta+2}{\theta-2}$ and then using (6) with exponent $q=\frac{\theta+2}{2}$, we have

$$
\int_{\mathbb{R}^{n}}|p|^{2}|u|^{\theta-2} d x \leq\|p\|_{L^{\frac{\theta+2}{2}}}^{2}\|u\|_{L^{\theta+2}}^{\theta-2} \leq C\|u\|_{L^{\theta+2}}^{\theta+2} .
$$

Similarly, we can bound

$$
\int_{\mathbb{R}^{n}}\left|\nabla p\left\|\left.u\right|^{\theta-1} d x \leq\right\| \nabla p\left\|_{L^{r}}\right\| u \|_{L^{(\theta-1) r^{\prime}}}^{\theta-1},\right.
$$

where again $r^{\prime}$ is conjugate to $r$. Choose $\theta$ so that

$$
(\theta-1) r^{\prime}=\theta+2,
$$

that is,

$$
\frac{1}{r}=1-\frac{1}{r^{\prime}}=1-\frac{\theta-1}{\theta+2}=\frac{3}{\theta+2}
$$

Then we have

$$
\theta=3 r-2 \geq 3 n-2>n,
$$

and we arrive at the estimate

$$
\begin{aligned}
\left(\int_{\mathbb{R}^{n}}|\nabla p \| u|^{\theta-1} d x\right)^{2 / 3} & \left(\int_{\mathbb{R}^{n}}|p|^{2}|u|^{\theta-2} d x\right)^{1 / 3} \\
& \leq\|\nabla p\|_{L^{r}}^{2 / 3}\|u\|_{L^{\theta+2}}^{\theta}=\|\nabla p\|_{L^{r}}^{2 / 3}\|v\|_{L^{2} \frac{\theta+2}{\theta}}^{2} .
\end{aligned}
$$

Note that (21) and (4) yield the equation

Choose

$$
s=\frac{2(\theta+2)}{3(\theta+2-n)} .
$$

$$
p=2 \frac{\theta+2}{\theta}, q=2 \frac{\theta+2}{n},
$$

satisfying (9). Observe that with this choice of $q$ the exponents $3 s / 2$ and $q / 2$ are conjugate. Absorbing the last term in (17) on the left of (8) and integrating in $t$, by Hölder's inequality with a constant $C_{1}=C_{1}(r)$ from (10) and (22) we then obtain

$$
\begin{aligned}
\|v\|_{L_{x, t}^{2, \infty}}^{2,}+\|v\|_{L_{x, t}^{p, q}}^{2} & \leq C\left(\left\|v_{0}\right\|_{L^{2}}^{2}+\int_{0}^{T}\|\nabla p\|_{L^{r}}^{2 / 3}\|v\|_{L^{p}}^{2} d t\right) \\
& \leq C_{1}\left(\left\|v_{0}\right\|_{L^{2}}^{2}+\|\nabla p\|_{L_{x, t}^{r, s}}^{2 / 3}\|v\|_{L_{x, t}^{p, q}}^{2}\right) .
\end{aligned}
$$


Since $s<\infty$, by absolute continuity of the Lebesgue integral for any $T_{0}<\infty$ we can find a uniform number $0<T<T_{0}$ such that $C_{1}\|\nabla p\|_{L_{x, t}^{r, s}}^{2 / 3}<1 / 2$ for any interval $J \subset\left[0, T_{0}\right]$ of length $T$. Covering $\left[0, T_{0}\right]$ with finitely many such intervals and iterating, from (24) we then obtain the bound

$$
\|v\|_{L_{x, t}^{2, \infty}}^{2,}+\|v\|_{L_{x, t}^{p, q}}^{2} \leq C\left\|v_{0}\right\|_{L^{2}}^{2}
$$

on $\left[0, T_{0}\right]$. Since $\theta>n$, we may again invoke Theorem 1.1 to conclude that $u$ extends smoothly to $\left[0, T_{0}\right]$.

\section{References}

[1] H. BEIRÃO DA VEIGA, Existence and asymptotic behavior for strong solutions of the NavierStokes equations in the whole space, Indiana Univ. Math. J. 36 (1987), 149-166.

[2] H. Beirão DA Veiga, A new regularity class for the Navier-Stokes equations in $\mathbb{R}^{n}$, Chinese Annals Math. 16 (1995), 407-412.

[3] H. Beirão DA VEIGA, Concerning the regularity of the solutions to the Navier-Stokes equations via the truncation method, in: Équations aux dérivées partielles et applications, 127-138, Gauthier-Villars, Éd. Sci. Méd. Elsevier, Paris, 1998.

[4] H. Beirão DA Veiga, A sufficient condition on the pressure for the regularity of weak solutions to the Navier-Stokes equations, J. Math. Fluid. Mech. 2 (2000), 99-106.

[5] L. C. Berselli and G. P. Galdi, Regularity criteria involving the pressure for the weak solutions of the Navier-Stokes equations, Proc. Amer. Math. Soc. 130 (2002), 3585-3595.

[6] L. Caffarelli, R. Kohn and L. Nirenberg, Partial regularity of suitable weak solutions of the Navier-Stokes equations, Comm. Pure Appl. Math. 35 (1982), 771-831.

[7] D. Chat and J. LeE, Regularity criterion in terms if pressure for the Navier-Stokes equations, Nonlin. Analysis, Ser. A 46 (2001), 727-735.

[8] L. Escauriaza, G. A. Seregin and V. ŠverÁk, $L^{3, \infty}$-solutions of the Navier Stokes equations and backward uniqueness, Russ. Math. Surv. 58 (2) (2003), 211-250.

[9] E. Fabes, B. Jones and N. M. Riviere, The initial value problem for the Navier-Stokes equations with data in $L^{p}$, Archive Rational Mechanics Analysis 45 (1972), 222-248.

[10] G. P. Galdi and S. Rionero, The weight function approach to uniqueness of viscous flows in unbounded domains, Arch. Rational Mech. Anal. 69 (1979), 37-52.

[11] Y. GigA, Solutions for semilinear parabolic equations in $L^{p}$ and regularity of weak solutions of the Navier-Stokes equations, J. Diff. Eq. 62 (1986), 186-212.

[12] E. Hopf, Über die Anfangswertaufabe für die hydrodynamischen Grundgleichungen, Math. Nachr. 4 (1951), 213-231.

[13] O. A. Ladyzhenskaya, The mathematical theory of viscuous incompressible flow, Gordon and Breach, rev. second edition, 1969.

[14] O. A. Ladyzhenskaya, Solution "in the large" of the nonstationary boundary value problem for the Navier-Stokes system in two space variables, Comm. Pure Appl. Math. 12 (1959), 427-433.

[15] J. Leray, Sur le mouvement d'un liquide visqueux emplissant l'espace, Acta Math. 63 (1934), 193-248.

[16] T. Ohyama, Interior regularity of weak solutions to the Navier-Stokes equations, Proc. Japan Acad. 36 (1960), 273-277.

[17] G. Seregin and V. Šverák, The Navier-Stokes equations and backward uniqueness, in: Nonlinear Problems in Mathematical Physics II, In honor of Professor O. A. Ladyzhenskaya, International Mathematical Series II (2002), 353-366.

[18] J. SERRIN, On the interior regularity of weak solutions of the Navier-Stokes equations, Arch. Rat. Mech. Anal. 9 (1962), 187-195. 
[19] H. Sohr, Zur Regularitätstheorie der instationären Gleichungen von Navier-Stokes, Math. Z. 184 (1983), 359-375.

[20] H. SoHr and W. VON WAHL, On the regularity of the pressure of weak solutions of NavierStokes equations, Archiv Math. 46 (1986), 428-439.

[21] V. A. Solonnikov, Estimates of the solutions of a nonstationary linearized system of Navier-Stokes equations, Amer. Math. Soc. Transl. 75 (2) (1968), 1-116.

[22] M. Struwe, On partial regularity results for the Navier-Stokes equations, Comm. Pure Appl. Math. 41 (1988), 437-458.

[23] S. TAKAHASHI, On interioir regularity criteria for weak solutions of the Navier-Stokes equations, manuscripta math. 69 (1990), 237-254.

[24] S. TAKahashi, On a regularity criterion up to the boundary for weak solutions of the Navier-Stokes equations, Comm. Partial Diff. Eq. 17 (1992), 261-285.

[25] W. von Wahl, Regularity of weak solutions of the Navier-Stokes equations, Proc. Symp. Pure Math. 45 (1986), 497-503.

[26] Y. Zhou, Regularity criteria in terms of pressure for the 3-D Navier-Stokes equations in a generic domain, Math. Annalen 328 (2004), 173-192.

[27] Y. Zhou, On regularity criteria in terms of pressure for the Navier-Stokes equations in $\mathbb{R}^{3}$, Proc. Amer. Math. Soc. (to appear).

[28] Y. ZHOU, On a regularity criterion in terms of the gradient of pressure for the Navier-Stokes equations in $\mathbb{R}^{N}$, preprint, 2004.

M. Struwe

Mathematik

ETH-Zürich

CH-8092 Zürich

Switzerland

(accepted: May 26, 2005; published Online First: September 6, 2006) 